\title{
A parametric study of occupational radiation dose in interventional radiology by Monte-Carlo simulations
}

Mahmoud Abdelrahmana,b,*, Pasquale Lombardoa, Anna Campc, Maria A. Duchc, Christophe Phillipsb, Alain Seretb, Filip Vanhaverea

a SCKCEN: the Belgian Nuclear Research Center, Boeretang 200, Mol 2400, Belgium

b ULiège: Cyclotron Research Centre, B30, 8 Allée du Six Août, Liège 4000, Belgium

c Institut de Tècniques Energètiques, Research Centre for Biomedical Engineering, Universitat

Politècnica de Catalunya, Barcelona, Spain

\begin{abstract}
This paper presents the results of a parametric study on the occupational exposure in interventional radiology to explore the influence of various variables on the staff doses. These variables include the angiography beam settings: $x$-ray peak voltage $(k V p)$, added copper filtration, field diameter, beam projection and source to detector distance. The study was performed using Monte-Carlo simulations with MCNPX for more than 5600 combinations of parameters that account for different clinical situations. Additionally, the analysis of the results was performed using both multiple and random forest regression to build a predictive model and to quantify the importance of each variable when the variables simultaneously change. Primary and secondary projections were found to have the most effect on the scatter fraction that reaches the operator followed by the effect of changing the $x$-ray beam quality. The effect of changing the source to image intensifier distance had the lowest effect.
\end{abstract}

\section{Introduction}

Fluoroscopically guided interventional procedures have been increasing around the world over the past 20 years [1]. These minimally invasive procedures substitute traditional surgery, resulting in reduced risk to the patient. In recent years, technological advancements have led to new development of diagnostic radiology in practice to provide high-quality images with optimization in radiation dose. However, the prolonged and frequent occupational exposure for interventional cardiologists/radiologists put them as one of the highest exposed group among medical staff, which can be high enough to cause concern [1-3]. In these exposure scenarios, staff is exposed to the scatter radiation field around the patient. Specifically, in unshielded situation, interventional radiologists are exposed to an inhomogeneous radiation field where the exposure is greatest below the table, less at the operator's waist level, and least at the eye level. The operator's eyes can be exposed to a significant dose in some situations (large patient, high-dose imaging protocol). In practice, protective shielding is often used such as lead aprons, table-mounted shields for lower body protection, or ceiling suspended shields. The magnitude and distribution of scatter radiation at the interventionalist position depends on various $x$-ray beam settings and imaging conditions. The radiation dose received by the interventionalist can vary for the same type of procedure and for similar dose to the patient [3], which shows the complexity of the scatter radiation field in practice. Several variables affect the distribution of doses at the area surrounding the patient. These variables include the angiography beam settings such as beam added filtration, field diameter, positioner rotations, etc. along with noncontrollable clinical situations such as patient abdomen thickness, position, and operator position. Evaluating the impact of each parameter is important to understand the occupational exposure in Interventional Radiology (IR).

The basic principle when selecting the $x$-ray beam settings in radiography is ensuring an appropriate image quality for the purpose of the procedure and, at the same time, the exposure should generate the lowest harm to the patient. From the image generation point of view, 
sufficient contrast and low noise are needed [4-6], which depend on the tissue to be observed (bone and soft tissues require different tube voltage) and the anatomical region to be explored (depending on the thickness of the body part examined tube voltage and tube current will be different). Tube voltage typically determines the maximum energy of the photons generated and, if it is high enough, the presence of characteristic x-rays (e.g. for a typical tungsten anode, $\mathrm{k}$ and $\mathrm{k}$ characteristic $\mathrm{x}$-rays appear around 59 and $69 \mathrm{keV}$ respectively). Adding filtration to the beam reduces the number of low-energy photons of thespectrum, thus increasing the mean energy of the resulting $\mathrm{x}$-ray beam.

As a principle of dose optimization to the patient, a minimum beam filtration $(2.5 \mathrm{~mm} \mathrm{Al}$ equivalent) is always required to attenuate very low energy $\mathrm{x}$-rays that are absorbed in the patient with minimal diagnostic benefit, but for some examinations additional filtration is also used. Subsequently, changing $\mathrm{kVp}$ or adding beam filtration changes the energy distribution of the scatter photons at the position of the medical staff. On the other hand, the geometrical configuration of the beam, determined by the beam diameter, the beam angular projection, and the source to detector distance, can also influence the penetration and the scattering around the patient. These geometrical parameters influence mainly the angular distribution of the scatter radiation. Although in interventional radiology (IR) some of these $x$-ray beam settings (e.g. tube voltage, total filtration) are automatically selected by an automatic exposure control system according to the medical procedure to be carried out and patient thickness, other parameters (angular projection, field size, source to detector distance) are selected by the medical staff. The goal of this paper is to quantify these influences, since the knowledge of them would help to reduce operator's doses. At present, personal dosimetry in IR is typically performed by issuing staff with physical dosemeters that give measurements of operational quantities, $\mathrm{Hp}$ (d), as an estimate of protection quantities, like the effective dose. Unfortunately, large uncertainties with measurements from current dosemeters exist especially in highly inhomogeneous radiation fields in IR. In ICRP publication 75 [7], it is stated that for personal dosimetry, the over-all uncertainty at the $95 \%$ confidence level in the estimation of effective dose around the relevant dose limit may well be a factor of 1.5 in either direction. This is certainly the case for IR workplaces, where the energy and the orientation of the radiation field are rarely known and can vary significantly. The use of protective garments in IR adds a lot of complexity to personal dosimetry because of complete or partial shielding of the organs. For example, practitioners use protective lead aprons as a common practice, which reduces doses significantly for the organs underneath. Currently, several methods have been proposed to estimate the effective dose when lead aprons are used, such as applying a correction factor to the dose measured by a $\mathrm{Hp}(10)$ dosemeter worn above or below the apron, or the use of a correlation combining the dose from a dosemeter worn above and another one worn below the lead apron. Nevertheless, it remains difficult to provide a conservative estimate with minimized overestimation of the effective dose under all possible exposure conditions [8].

On the other hand, computational dosimetry has evolved rapidly in the past decades. The use of the Monte-Carlo (MC) method in radiation dosimetry has increased exponentially. This technique allows the simulation of the energy, position and direction of the particles. Hence, absorbed dose and other dosimetric quantities like fluence, kerma, etc. can be calculated directly. Although computer power restricted early applications to simple geometries, like solid prisms or cylinders, the availability of increased computing power nowadays allows the simulation of complex 3-D geometries like a detailed anthropomorphic human body phantom. Schueler et al. (2006) [9] conducted an experiment mimicking various clinical imaging conditions and using a patient-equivalent abdomen physical phantom. The study investigated how operator exposure in interventional radiology is affected by various common fluoroscopic imaging conditions. In particular, the effect of changing the field of view and the effect of adding copper filters; among other clinical conditions like increasing patient abdomen thickness and 
changing patient position. The experiment investigated the effect of changing those parameters by measuring air kerma rates at the collar and waist locations of the operator and at the entrance of the patient phantom. Although the study gave an outlook

on assessing occupational exposure in interventional radiology, to examine the effect of all possible parameters experimentally can be impractical. While, it is necessary to examine several imaging conditions as in clinical practice many of those parameters change simultaneously. Within the ORAMED project, the studies of Koukorava et al. (2011) [10] and Carinou et al. (2011) [11] examined the effect of some parameters separately on the dose to the extremities and eye lens which was possible only through Monte-Carlo simulations. Specifically, the effect of beam projections, field size, and beam quality was studied for two typical positions of the operator simulating femoral and radial coronary access. Similarly, recent studies of Ferrari et al. (2018) [12] and Ferrari et al. (2019) [13], within the activities of EURADOS Working Group 12, evaluated the exposure condition of the medical staff in few selected interventional radiology procedures using Monte-Carlo simulations. Unlike $[10,11]$, the study evaluated simulated $\mathrm{Hp}(10)$ and effective dose rather than extremities and eye lens; and comparing the results with measurements within the study and from the study of Olgar et al. [14]. Yet, the study was limited to few cases of changing some projections and beam qualities. The study of Badal et al. (2013) [15] and Abdelrahman et al. (2020) [16] presented a dose monitoring system based on accurate $\mathrm{MC}$ simulations and 3D localization system that can be used to calculate doses for both patient and staff in IR. Although Monte-Carlo simulations of occupational doses in IR were proven as feasible, those simulations are computationally expensive and require some effort to obtain all the different input parameters. The CONCERT-funded project PODIUM (Personal Online Dosimetry using Computational Methods) [17] aimed

to provide real-time, online assessments of effective doses for occupational exposures, by tracking the motion of individuals in the workplace fields. The project investigated two different approaches to provide online monitoring of occupational doses: i) accelerated Monte-Carlo simulations, and ii) an intermediate approach based on the creation of look-up tables comprising information of the radiation field and the organ dose conversion coefficients for phantoms of different statures and postures. Due to the large number of variables that contribute to the dose of the IR staff, the size of such set of dose conversion coefficients, to take into account every possible combination of variables, can be extensive. To set-up a look-up table of dose conversion coefficients that can be used practically for personal dose assessment in IR, a sensitivity analysis to study quantitatively the influence of each parameter

separately was performed. The results of the present paper can thus also be used to facilitate such computational dosimetry. The main goal of our study is to extend the examination not only to take into account more variables that contribute to the dose, but also to the combinations of these variables over the operating range of fluoroscopy and angiography procedures. In the present study, the examination of different combinations of parameters is focused on the evaluation of the dose levels at the location of the whole body dosemeter.

For that purpose, a series of Monte-Carlo simulations were performed. In total, we did the analyses for 5628 different combinations of parameters. A step further is to use the results of the simulations to build a model by a regression analysis that describes the dose for different combination of parameters within the range of simulated cases. This model could be used to quantify the importance of each variable on the dose. Regression analysis [18] is a powerful statistical method to examine the relationship between one or more dependent variables and independent variable(s).

\section{Materials and methods}

In interventional radiology procedures, the main elements of the exposure situation are the patient who is exposed to the primary beam, the interventionalist who is mainly exposed to the scatter radiation, the operating table and the image intensifier. Thus, estimation of the personal dose equivalent to the interventionalist requires the use of a model representing the patient 
lying on the surgical table under an image detector. Besides the geometrical aspects, the angiographic beam can be simulated with an $\mathrm{x}$-ray spectrum located at the position of the xray tube and scaled according to the field of view with a conical shape. The generation of the $x$-ray spectra in tungsten anodes, which is the most common types of anodes in IR set-ups, is performed using validated models found in literature $[19,20]$.

The characteristics of the xray tube taken into account to generate the beam spectra were the following:

- Anode material: Tungsten

- Anode angle: 12 은

- Minimum filtration: $2.5 \mathrm{~mm}$ Al.

Monte-Carlo simulations were performed using MCNPX v2.7 [21]. MCNPX is a general-purpose Monte-Carlo radiation transport code that tracks nearly all particles at nearly all energies. The approach that was used for the calculation of the staff dose in MCNPX is presented in the following sections. In MCNPX, the reliability of results are assessed based on statistical tests it performs on the tally. One of the most important is the relative error R.1 R generally must be less than $10 \%$ for meaningful results [22]. Thus, the statistical uncertainties in our simulations were limited to relative error $R<10 \%$. Each studied parameter was set within an operating range found in literature for fluoroscopy and angiography procedures [23]. These typical radiation field characteristics in interventional radiology and cardiology was also supported by statistics from the two hospitals participating in PODIUM (Skåne university hospital in Malmö and St.James's hospital in Dublin).

The parameters that were studied are the following:

- The x-ray peak tube voltage: $70,80,90,100,110$, and $120 \mathrm{kVp}$.

- The beam projections: Anterior (also called Postero-Anterior (PA)), Left Anterior Oblique (LAO) and Right Anterior Oblique (RAO) at the angles of $15^{\circ}, 30^{\circ}, 45^{\circ}, 60^{\circ}$, and $75^{\circ}$, Caudal (CAU) and Cranial (CRA) projections at $15^{\circ}$, and $30^{\circ}$ as well as combinations of these projections.

- The field size: 20,25 , and $30 \mathrm{~cm}$ diameter.

- The source to image intensifier distance (SID): 100,110 , and $120 \mathrm{~cm}$

- The beam quality: without added filtration and with added copper filtration of $0.1,0.2$, $0.3,0.6$, and $0.9 \mathrm{~mm}$ thickness.

\subsection{Geometry}

A representation of a typical geometrical set-up in IR is shown in Fig. 1. In this set-up, the physician/main operator is represented with the Realistic Anthropomorphic Flexible (RAF) phantom [24]. However, for the purpose of the present study and for only calculating the dose at the location of a dosemeter, no phantom was introduced for the operator in the simulations. Also, the patient phantom was simplified as a slab phantom. This simplified geometry was used to optimize the simulation run time, which allowed the extension of the number of simulations that could be achieved. At the same time, these simplifications should not compromise the validity of the model. For that reason, the approximated geometry for the MC simulations was validated against measurements during the PODIUM project as reported in [25]. Fig. 2 shows the geometry used in MCNP. The patient was modeled as a prism of $100 \mathrm{~cm}$ length, $32 \mathrm{~cm}$ width and $23 \mathrm{~cm}$ thickness, and composed of the ICRU tissue. 2 The image intensifier was simulated by a squared layer of $0.3 \mathrm{~cm}$ of lead. The patient's tabletop and mattress were simulated as a layer of $2.5 \mathrm{~mm}$ of aluminum to account for their equivalent attenuation.

\subsection{Tallying and dose calculation}


To calculate the absorbed dose that approximate the personal dose equivalent $\mathrm{Hp}(10)$, the tally F6 was used to score the energy deposition in MeV/g per particle in a volume that is behind 10 $\mathrm{mm}$ tissue equivalent volume as described in Figure 2. This volume is placed facing the patient phantom on the right side.

This tally estimates energy deposition by integrating the track-length photon flux weighted by photon heating numbers according to Equation 1. These numbers represent the average kinetic energy given to electrons along the photon path. Therefore, this tally is approximately valid only when most of the electrons are trapped in the tallied cells, which is the case for energy range up to $120 \mathrm{keV}$. The tallied volume is located at a typical position of the operator at $(52.5 \mathrm{~cm}, 20$ $\mathrm{cm}$, and $49.5 \mathrm{~cm}$ ) relative to the iso-center as the origin as shown in Figure 1. This position is considered for femoral or radial access procedures; and it was obtained as the most frequent location through several recordings using motion-tracking system as described in Error! No s'ha trobat l'origen de la referència.. The position is also in agreement with data from literature in the study of Schueler et al. (2006) Error! No s'ha trobat l'origen de la referència. and Principi et al. Error! No s'ha trobat l'origen de la referència.

$$
\mathrm{F} 6=\frac{\rho_{\mathrm{a}}}{\mathrm{m}} \int \mathrm{dE} \int \mathrm{dt} \int \mathrm{dV} \int \mathrm{d} \Omega \sigma_{\mathrm{t}}(\mathrm{E}) \mathrm{H}(\mathrm{E}) \Psi(\overrightarrow{\mathrm{r}}, \widehat{\Omega}, \mathrm{E}, \mathrm{t})
$$

Where $\rho_{\mathrm{a}}$ is the atomic density (atoms/barn-cm), $\mathrm{m}$ is the cell mass (g), $\mathrm{E}$ is the energy (MeV), $\mathrm{t}$ is the time (sh), $\mathrm{V}$ is the volume $(\mathrm{cm} 3), \Omega$ is the direction vector, $\sigma_{\mathrm{t}}$ is the microscopic total cross section (barns), $\mathrm{H}(\mathrm{E})$ is the heating response in $\mathrm{MeV} / \mathrm{g}, \Psi$ is the angular flux and $\Psi(\overrightarrow{\mathrm{r}}, \widehat{\Omega}, \mathrm{E}, \mathrm{t})$ is in (particles/cm2/sh/MeV/steradian).

This calculated absorbed dose at the operator position is given per particle. Since all calculated doses are given per simulated particles and thus, they need to be normalized to a reference value. This reference value is the kerma area product (KAP) which is experimentally measurable and is reported in the radiation dose structure report (RDSR) defined by DICOM (DICOM PS3.16 2020b) [23], the most commonly used method of recording and storing dose data. In the simulations, absorbed dose was tallied in a thin layer of air located at the KAP meter location (20 cm from the source), scaled to cover completely the beam depending on the $x$-ray field size, and rotating together with the source and the image detector.

In particular, the following ratio was calculated:

Ratio $\left(\frac{\text { Operator }}{\text { Reference }}\right)=\mathrm{R}\left(\frac{\mathrm{Sv}}{\mathrm{Gy}}\right)=\frac{\text { Absorbed Dose at } 10 \mathrm{~mm} \text { depth at the operator position }}{\text { Absorbed Dose in air at the KAP meter location }}$

\subsection{Multiple regression analysis}

The purpose of the regression analysis is to build a machine learning model that is trained using the results of the simulated cases, test its accuracy, and finally obtain the feature importance score for each studied parameter on the dose. There are various techniques of regression analysis available. The selection of the adequate technique depends mainly on the number of independent variables, the type of the data and the shape of the regression line. The advances and availability of machine learning algorithms make it easier to build a multi-variable complex relationship model. Two different regression analysis methods were applied to the results of the simulations: multiple polynomial and random forests regression analysis. While multiple polynomial regressioncan be used to build a predictive model that allow the user to examine the dose for different combination of parameters using a simpler polynomial equation, it cannot yield quantitative estimate of the importance of each dependent variable on the predictor. 


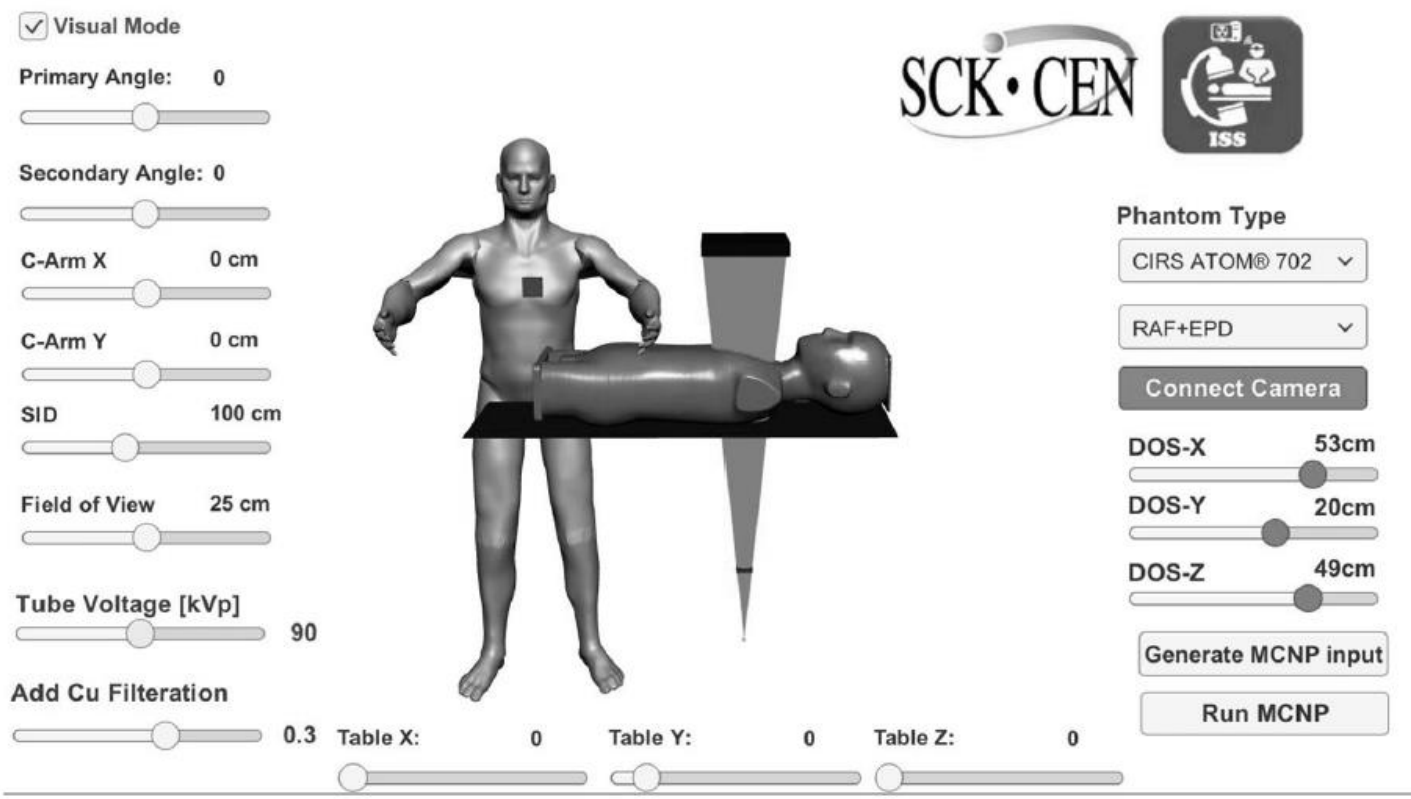

Fig. 1. Representation of the geometrical set-up in IR including the phantoms for the physician and the patient.

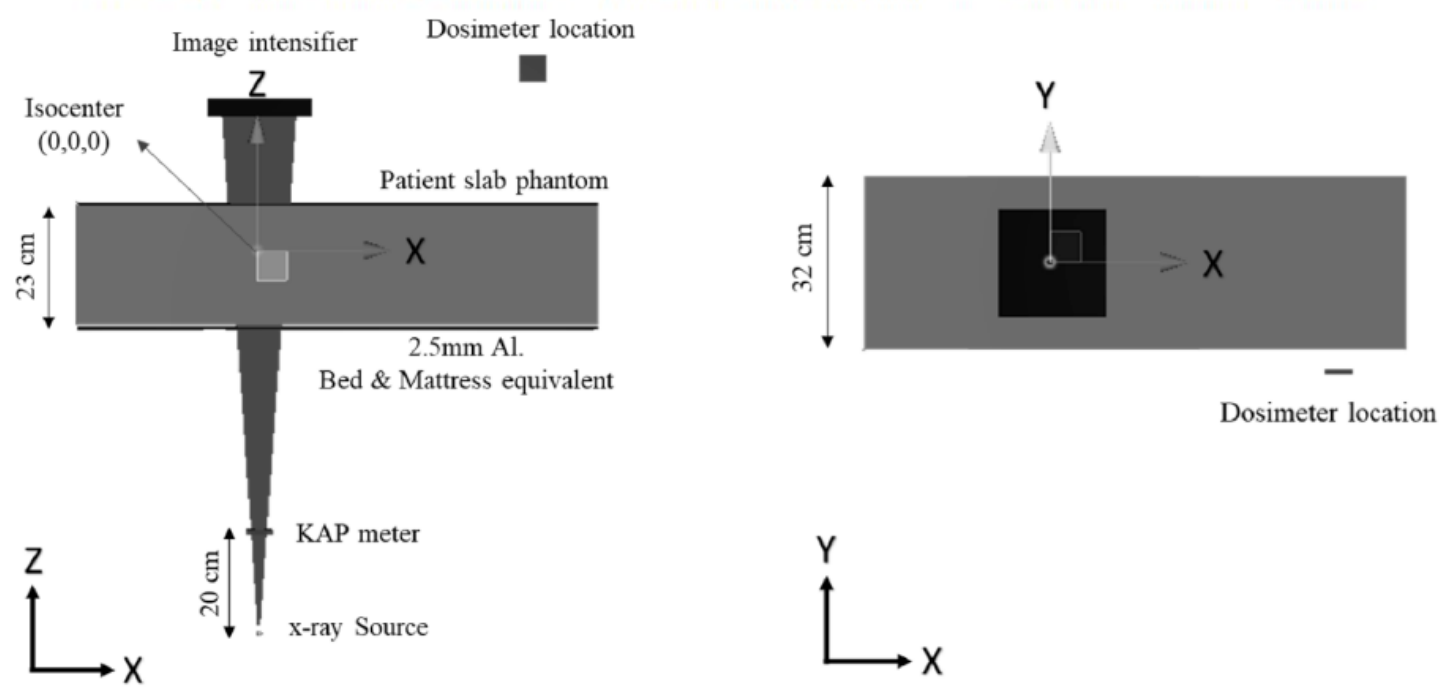
(a) Front-view
(b) Top-view

Fig. 2. Simplified geometry used in MCNPX simulations.

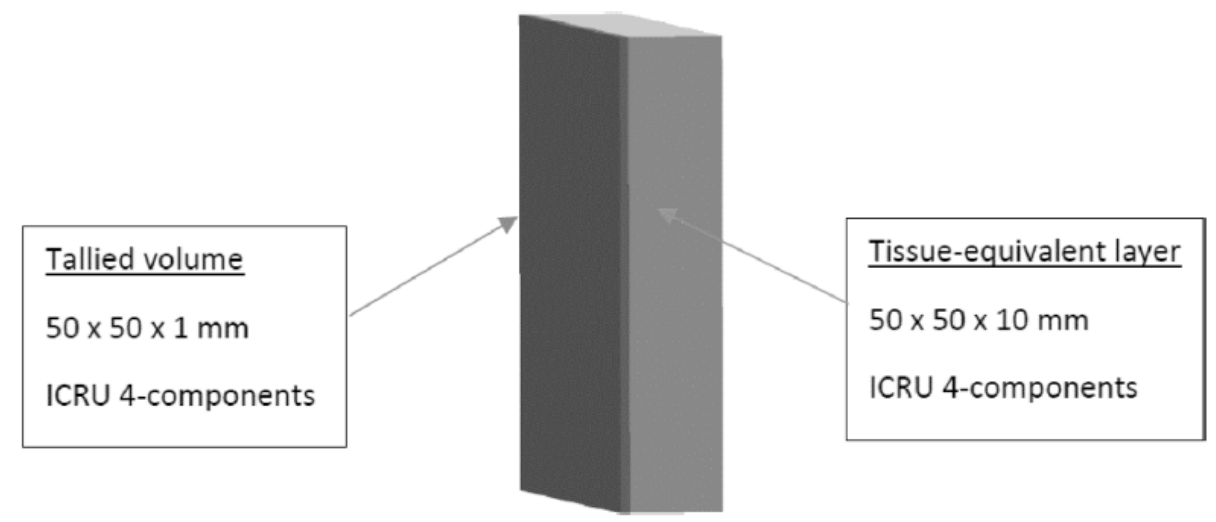

Fig. 3. Representation of the tallied volume in MCNPX. 

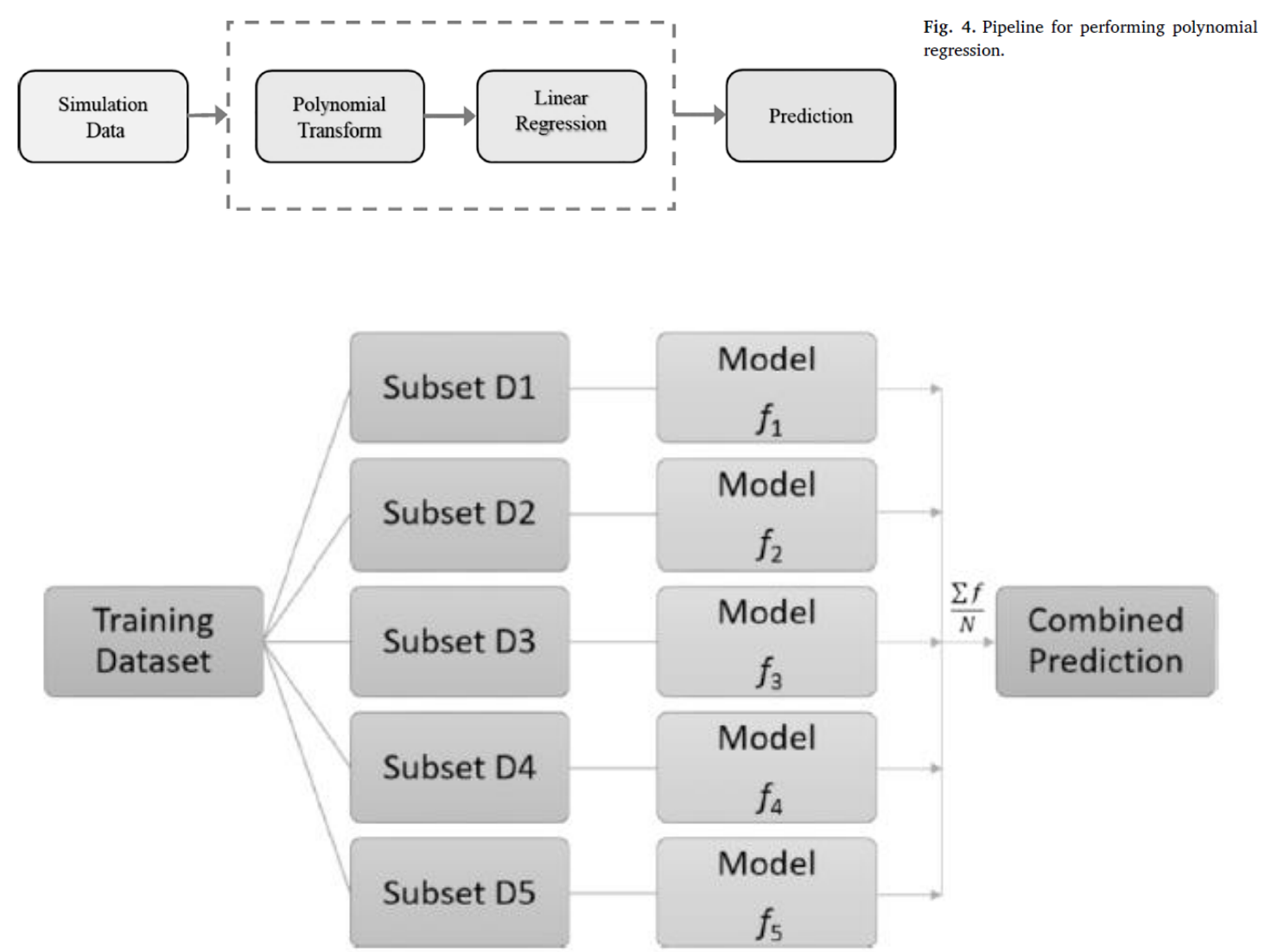

Fig. 5. Simplified representation of model ensembling approach used in random forests.

Thus, random forest regression was also used for its feature importance function.

\subsubsection{Polynomial multiple regression}

The general form of a complete $n$-degree polynomial regression model in one variable $X$ modelled as:

$$
y=\beta_{0}+\beta_{1} X+\beta_{2} X^{2}+\beta_{3} X^{3}+\cdots+\beta_{n} X^{n}+\varepsilon
$$

Where $y$ is the dependent variable, betas are the regression coefficients for different $n$th powers of the independent variable $X$ and $\varepsilon$ is the residual error. With the increasing degree of the polynomial, the complexity of the model also increases. Therefore, the value of $n$ must be chosen precisely. If this value is low, then the model will not be able to fit the data properly and if high, the model may overfit the data. Our model was build using the implementation of polynomial regression in Scikit-learn [24]. The function correlates six independent variables (X1 $-\mathrm{X6})$ to the dependent variable $(\mathrm{Y})$ where:

$\mathrm{X} 1$ : X-ray tube peak voltage $(\mathrm{kVp})$

$\mathrm{X} 2$ : positioner primary angle in degrees (positive for $\mathrm{LAO}$ and negative for RAO projections)

$\mathrm{X} 3$ : positioner secondary angle in degrees (positive for CRA and negative for CAU projections)

$\mathrm{X}$ : added copper filtration thickness in $\mathrm{mm}$

$\mathrm{X} 5$ : field diameter in $\mathrm{cm}$ 
X6: source-to-image intensifier distance (SID) in $\mathrm{cm}$

Y: R (Sv/Gy)

The implementation of polynomial regression is a two-step process as described in Fig. 4. First, we transform our data into a polynomial using the PolynomialFeatures function from Scikit-learn and then use linear regression to fit the parameters:

To determine the degree of the polynomial that yield the optimum accuracy, the model accuracy was evaluated over several degrees of polynomial up to the 10th degree. RMSE (Root Mean Squared Error) is one of the most popular evaluation metric used in polynomial regression problems. It follows an assumption that errors are unbiased and follow a normal distribution. RMSE can be calculated as the following:

$$
R M S E=\sqrt{\frac{\sum_{i=1}^{N}\left(\text { Predicted }_{i}-\text { Actual }_{i}\right)^{2}}{N}}
$$

Where, $\mathrm{N}$ is total number of simulated cases.

It is concluded that the 3rd degree (cubic) polynomial regression yields the optimum accuracy of the model with the least RMSE.

\subsubsection{Random forests regression}

Random forests are among the most popular machine learning methods to model complex dependencies thanks to their relatively good accuracy, robustness and ease of use [29]. The algorithm is an ensemble method that aggregates the predictions of multiple regression trees for decisions. In decision trees, every node is a condition of how to split values in a single feature (i.e. one of the studied parameters that affect the dose), so that similar values of the dependent variable (i.e. the dose) end up in the same set after the split. Node condition is based on impurity, which for regression trees is its variance. The impurity decrease from each feature can be averaged over different trees and the features are then ranked according to their contribution. Identifying which variables are important can help in the optimization of the dose to the staff by either avoiding, if possible, certain combinations of parameters that lead to higher doses.

Mathematically, the random forest algorithm is a type of additive model that predicts by combining decisions from a set of base models; and hence, this group of models can be described as:

$F(x)=f 0(x)+f 1(x)+f 2(x)+\ldots$

Where the final model $\mathrm{F}$ is the sum of simple base models $\mathrm{fi}$.

This broad technique of using multiple models to obtain better predictive performance is called model ensembling. In random forests, all the base models are constructed independently using a different subsample of the data as illustrated in Fig. 5. Fortunately, many implementations of this ensembling random forest models are available. In this study, the implementation available in Scikit-learn [28] was used.

After obtaining the results of the simulations, the dataset for a regression analysis using random forests algorithm can be prepared. The dataset contains the studied parameters as features (Xn) and the corresponding dependent variable ( $\mathrm{y}$ ) as the ratio $\mathrm{R}$ calculated by Eq. (2).

This dataset was then split into a training and testing datasets. The training dataset is the data, which is used to build the model, while the testing dataset is the set of data with which the resulted model can be tested. We followed the classical approach of $80 / 20 \%$ training/testing 
split which is used often in machine learning, and it is based on Pareto principle [30]. Any bias that can results from the random selection of the test dataset should be reflected in the statistical metrics of the resulted model, and in particular in the Mean Squared Error (MSE). 3 Inour case, the process of the split was performed over multiple folds until the model reached a good overall accuracy. In this case, $20 \%$ of the results were used for testing the model. The overall accuracy of the model can be calculated with the coefficient of determination4 (R2). The higher the model accuracy, the better the estimation of the importance coefficients is.

Finally, for regression, the feature importance in Scikit-learn is the variance reduction, which can be calculated with

Mean Absolute Error (MAE): $\frac{1}{N} \sum_{i=1}^{n}\left|y_{i}-\mu\right|$ and $\mu$ is the mean given by: $\frac{1}{N} \sum_{i=1}^{n} y_{i}$. The sum of all feature importance coefficients is 1 such that the higher the coefficient, the more important the feature is.

\section{Results}

First, the effect of changing each parameter independently was studied. In the following sections, the magnitude by which each parameter affects the dose to the operator is presented. Moreover, it is also important to study the effect of the parameters when combined which is concluded by the results of the regression analysis.

\subsection{Effect of changing $x$-ray peak tube voltage $(k V p)$}

Fig. 6a shows the changing of the peak voltage of the x-ray tube on the operator's dose, while keeping the other beam parameters constant. Without added filtration, the operator's dose is quite dependent on $\mathrm{kVp}$, and a linear equation can describe the trend. For the specific condition studied, changing $\mathrm{kVp}$ from 70 to 120 , the dose was multiplied by approximately three and half as shown in Fig. 6b. This is an expectedoutcome as the increase of the $k V p$ increases the maximum energy of the radiation beam. Therefore, this increases both the penetration power of the $x$-rays as well as the scatter radiation around the patient.

\subsection{Effect of beam quality: added Copper filtration}

The present study examined the effect of six different cases of beam filtration: without added filters, with $0.1 \mathrm{~mm}, 0.2 \mathrm{~mm}, 0.3 \mathrm{~mm}, 0.6 \mathrm{~mm}$, and $0.9 \mathrm{~mm}$ of added copper filters. As the beam filtration is simultaneously changing with $\mathrm{kVp}$ in practice, it is useful to show the effect of added filtration on the dose while changing the $\mathrm{kVp}$. Fig. 7a shows that the dose increases sub-linearly with the thickness of the copper filter. At lower $k V p$, the increase in the dose by adding copper filtration is greater than the increase at higher $k V p$ as shown in Fig. 7b. The beam hardening, as a consequence of the additional copper filtration, leads to a higher dose to the operator as the $\mathrm{kVp}$ increases. Consequently, the dose can increase by up to a factor 4.3 at $70 \mathrm{kVp}$ by adding copper filters; while at $120 \mathrm{kVp}$, the multiplicative factor goes only up to 2.6. It is important to highlight that in practice the change of the beam quality is also combined with a reduction in the measured KAP, as such when normalizing the results of the Monte-Carlo simulations, the dose for both the patient and the operator can be reduced. 


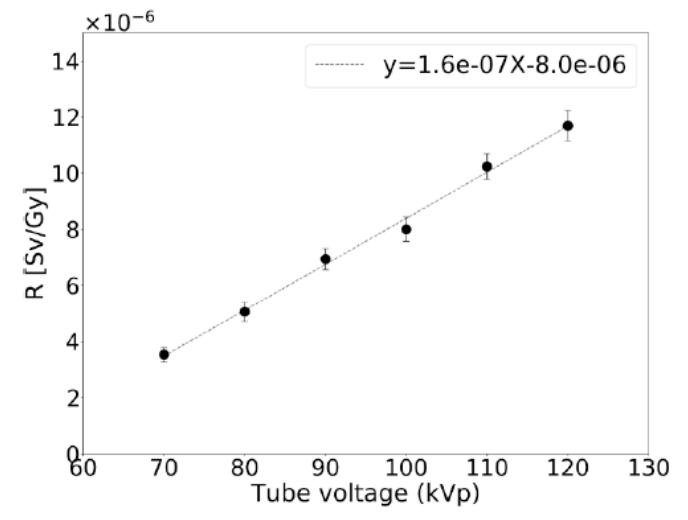

(a) Non-normalized results

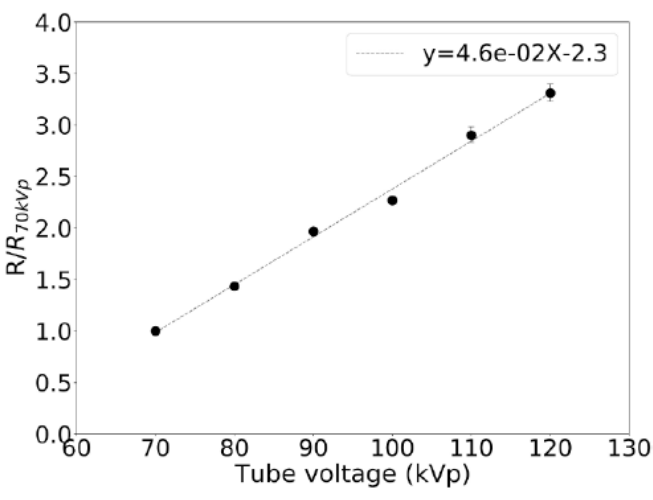

(b) Normalized results by $70 \mathrm{kVp}$

Fig. 6. $R(\mathrm{~Sv} / \mathrm{Gy}) \mathrm{vs} \mathrm{kVp}$ with no added filtration, PA projection, $20 \mathrm{~cm}$ field diameter, and $100 \mathrm{~cm}$ SID.

\subsection{Effect of beam projection}

Fig. 8 shows the variation of the operator dose for different beam primary projections at several beam energies. The operator dose is strongly influenced by the primary rotation angle i.e. Left Anterior Oblique (LAO) and Right Anterior Oblique (RAO) projections. However, within the region of $45^{\circ} \mathrm{RAO}-30^{\circ} \mathrm{LAO}$, it is reasonable to approximate the trend with a linear interpolation.

For secondary projections, the dose trend in Fig. 9a shows a plateau for secondary projections between 0 and $30^{\circ}$ Cranial where the dose slightly changes within 10-40\%, while, the dose is declining sharply against secondary angles beyond $15^{\circ}$ caudal. The lowest dose is observed at $30^{\circ}$ caudal and the maximum at $15^{\circ} \mathrm{cranial}$ and hence the dose can increase up to slightly 8 times as shown in Fig. 9b. In all cases, the dose is also higher for LAO or CRA projections than for RAO or CAU projections. This is due to the shielding effect by either the patient or the image intensifier when using RAO or CAU projections. For this specific location of the dosemeter, the dose decreases to their lowest value at $45^{\circ}$ RAO before it starts to increase again for angles higher than $45^{\circ}$ that is when the image intensifier is no longer shielding the dosemeter.

The study also examined the effect of changing simultaneously both the primary and secondary projections. The effect of changing simultaneously the positioner primary and secondary angles is presented in Figs. 10 and 11 for 70 and $110 \mathrm{kVp}$. LAO projections higher than 30 degrees when combined with any CRA projection seem to increase substantially the dose to the operator. On the other hand, combining CAU projection with any LAO projection decreases significantly the dose. We observed the highest dose to occur at $75^{\circ} \mathrm{LAO}$ combined with $30^{\circ} \mathrm{CRA}$ while the lowest dose is found to be at $30^{\circ} \mathrm{RAO}$ combined with $30^{\circ} \mathrm{CAU}$.

\subsection{Effect of changing field size}

Three field sizes of 20,25, and $30 \mathrm{~cm}$ diameter of the conical beam were studied. These field sizes correspond to circular field areas of $314,490,707 \mathrm{~cm} 2$, respectively projected at the image intensifier. Fig. 12a shows that the dose, for this specific configuration, is directly proportional to the field size for all tube voltages, which is found in agreement with the experimental data from Marshal et al. (1992) [31]. For all beam qualities, the doses are higher when a larger field size is used as more scatter radiation is produced when a larger primary beam intersects the patient. The dose increase is greater at higher $k V p$ compared to lower $k V p$ as shown in Fig. 12b. Increasing the field diameter from $20 \mathrm{~cm}$ to $30 \mathrm{~cm}$, multiplies the dose by a factor up to 2.5 . 


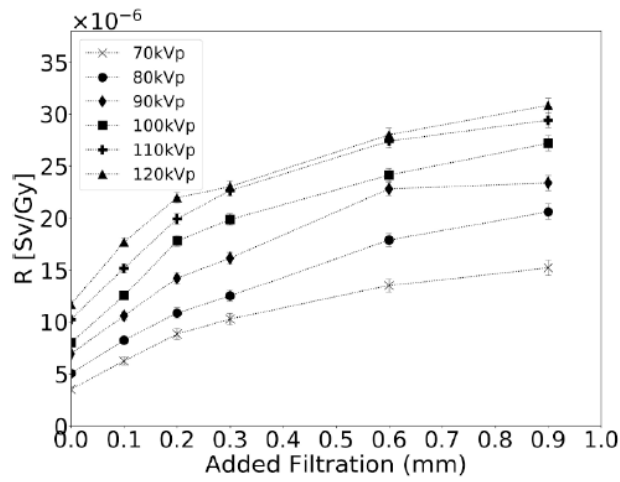

(a) Non-normalized results

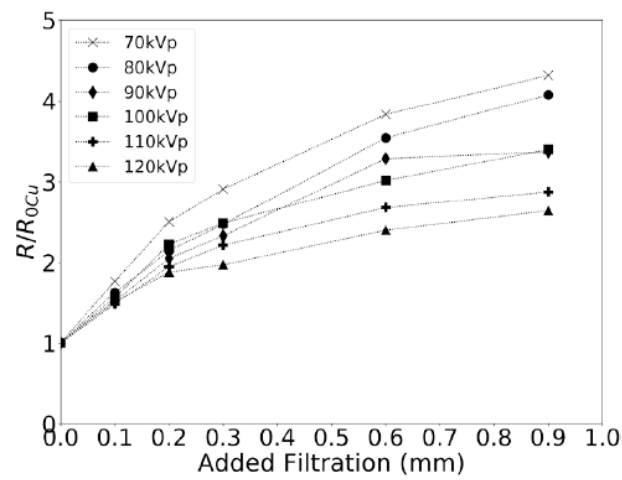

(b) Normalized results by no added

filtration

Fig. 7. $R(\mathrm{~Sv} / \mathrm{Gy})$ vs added copper filtration in $\mathrm{mm}$ for different $\mathrm{kVp}$ with PA projection, $20 \mathrm{~cm}$ field diameter, and $100 \mathrm{~cm}$ SID.

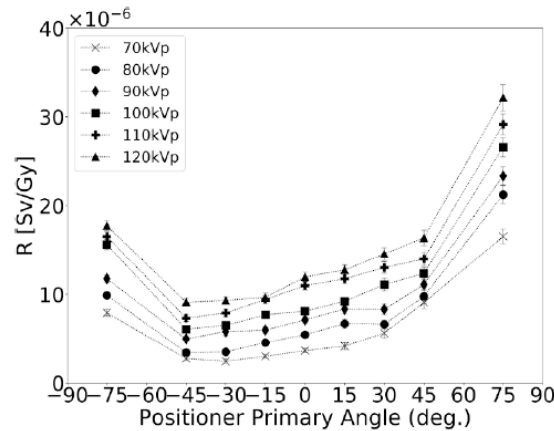

(a) Non-normalized results

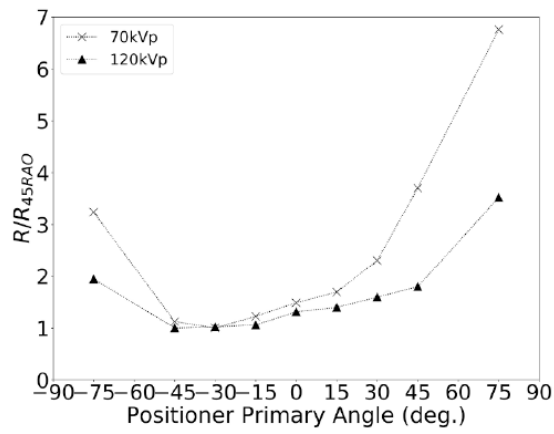

(b) Normalized results by $45 \mathrm{RAO}$

Fig. 8. $R(\mathrm{~Sv} / \mathrm{Gy})$ vs positioner primary angle of irradiation, for different $\mathrm{kVp}$ with no added copper filtration, $20 \mathrm{~cm}$ field diameter, and $100 \mathrm{~cm}$ SID.

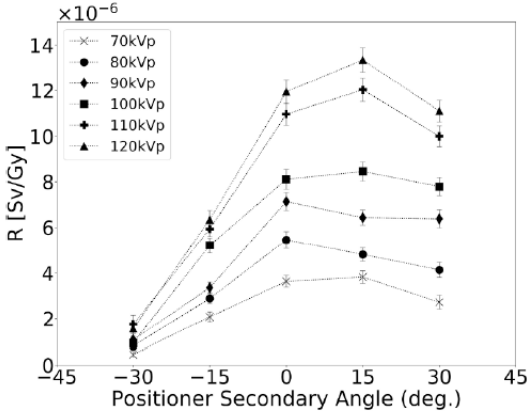

(a) Non-normalized results

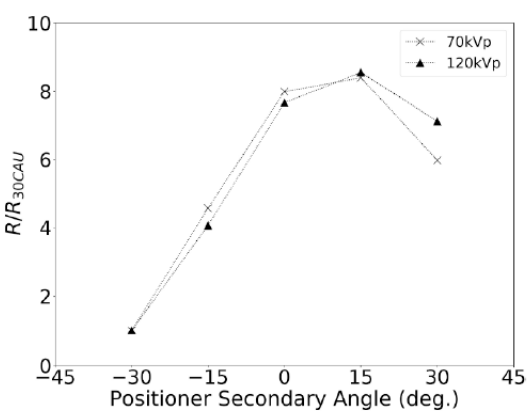

(b) Normalized results by 30CAU

Fig. 9. $R$ (Sv/Gy) vs positioner secondary angle of irradiation, for different $\mathrm{kVp}$ with no added copper filtration, $20 \mathrm{~cm}$ field diameter, and $105 \mathrm{~cm}$ SID

\subsection{Effect of changing source-to-Image Intensifier distance}

Typically, the source to detector distance (SID) changes for the purpose of magnification and hence it is linked to the field of view as well. Without added collimation and assuming the distance between the source and the isocenter is fixed, the field area decreases when the SID increases. Similar to the change in the field diameter, increasing the distance between the source and the image intensifier increases slightly the dose at the dosimeter position. However, in practice, when the magnification is increased, the radiation dose has to be increased in order to compensate for a smaller area of anatomy. In our simulations, the distance between the source and the patient was fixed which means the distance left between the patient and the 
detector increased. We studied the effect of increasing the SID on the scatter fraction $\mathrm{R}$ and the ratio of R over the projected beam area on the KAP meter (Sv/Gy. cm2). At lower kVps, increasing the source to image intensifier distance from $100 \mathrm{~cm}$ to $120 \mathrm{~cm}$ has negligible effect on the dose to the operator, while for $\mathrm{kVpsequal}$ to $120 \mathrm{kV}$, the scatter fraction $\mathrm{R}$ decreases by $15 \%$ while the ratio(Sv/Gy. $\mathrm{cm} 2$ ) increases by approximately $20 \%$ as shown in Fig. 13 . Fixing the field of view at $20 \mathrm{~cm}$ diameter and increasing the SID, the scatter

fraction $\mathrm{R}$ slightly decreases. This is due to the decrease in the field area intersecting the patient and the KAP meter. While, the absorbed dose at the dosimeter location slightly increases as a result of increasing the distance from the patient to the image detector that allows scatterd radiation to reach the dosimeter location. In clinical situations, the change of SID is usually accompanied by changes to the other parameters, but ingeneral, it is always desirable to minimize the distance between the patient and the image intensifier to reduce the dose to the staff.

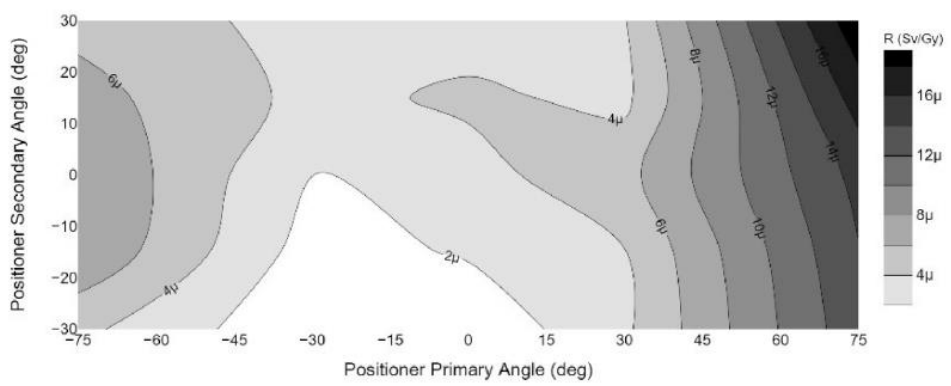

(a) Distribution at $70 \mathrm{kVp}$

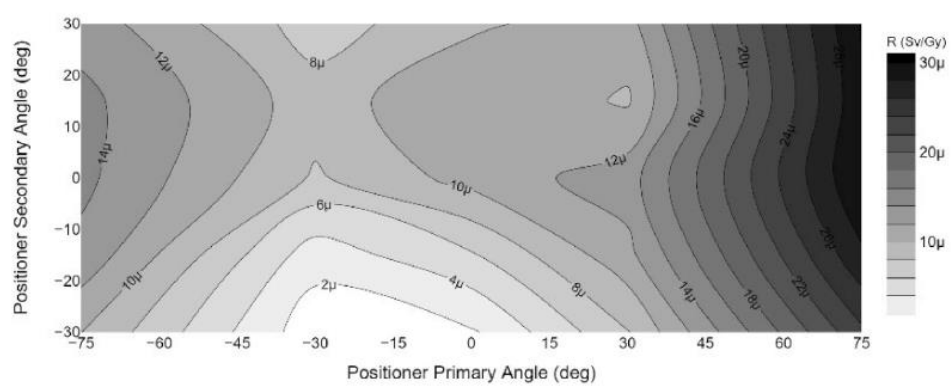

(b) Distribution at $110 \mathrm{kVp}$

Fig. 10. Distribution of $R$ (Sv/Gy) over positioner primary and secondary angle combination, with no added copper filtration, $20 \mathrm{~cm}$ field diameter, and $110 \mathrm{~cm}$ SID.

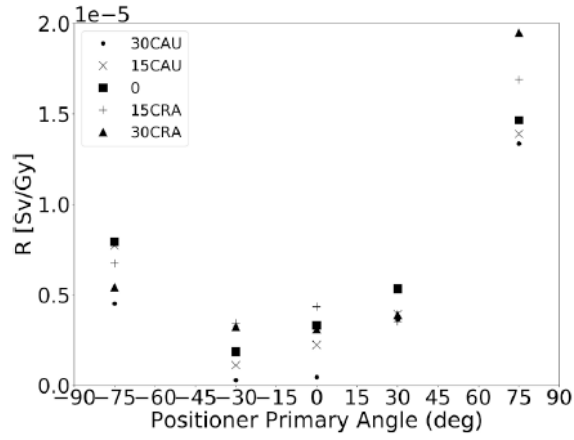

(a) At $70 \mathrm{kVp}$

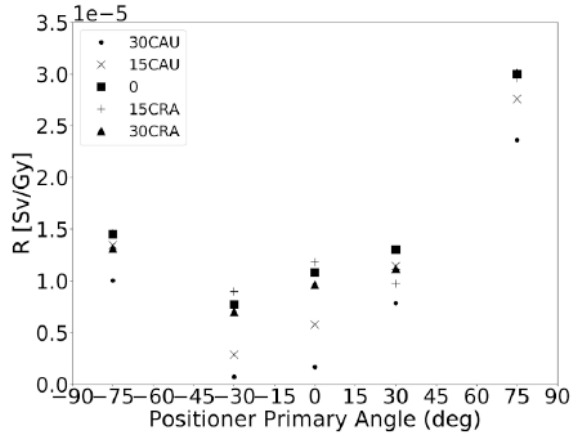

(b) At $110 \mathrm{kVp}$

Fig. 11. $R(\mathrm{~Sv} / \mathrm{Gy})$ vs positioner primary and secondary angle combination, with no added copper filtration, $20 \mathrm{~cm}$ field diameter, and $110 \mathrm{~cm}$ SID. 


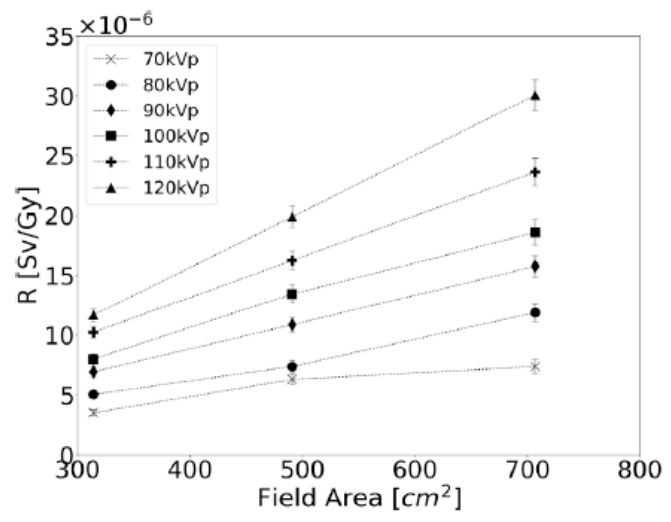

(a) Non-normalized results

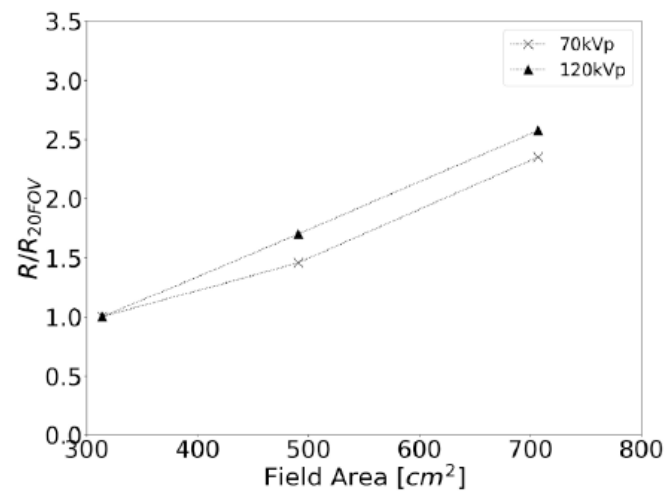

(b) Normalized results by $20 \mathrm{~cm}$ field diameter

Fig. 12. $R(\mathrm{~Sv} / \mathrm{Gy})$ vs field area $\left(\mathrm{cm}^{2}\right)$ for different $\mathrm{kVp}$ with no added copper filtration, PA projection, and $100 \mathrm{~cm}$ SID.

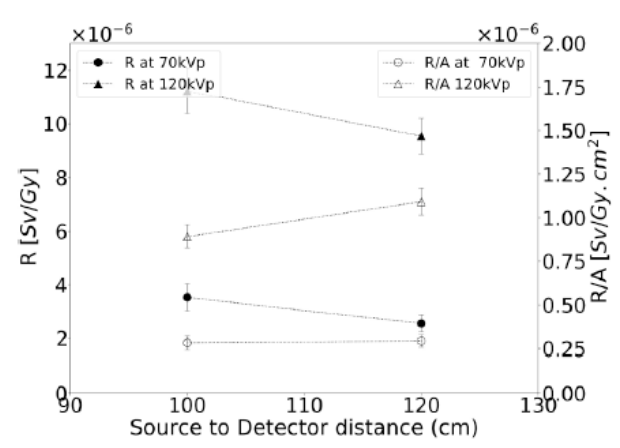

(a) Non-normalized results

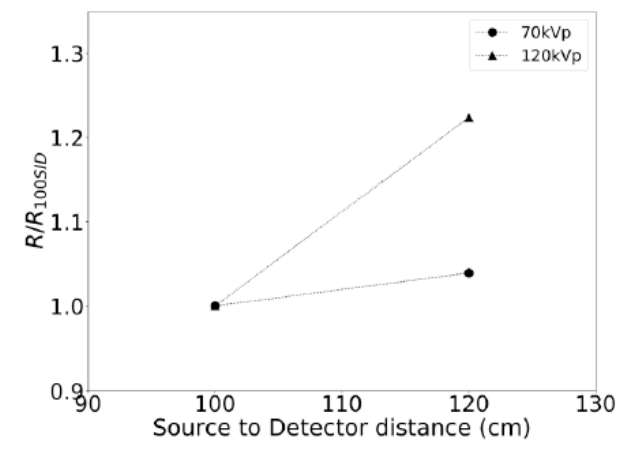

(b) Normalized results by $100 \mathrm{~cm}$ SID

Fig. 13. $R(S v / G y)$ and $R / A\left(S v / G y . \mathrm{cm}^{2}\right)$ vs SID, for 70 and $120 \mathrm{kVp}$, with no added copper filtration, $20 \mathrm{~cm}$ field diameter, and PA projection.

\subsection{Independent effect of each parameter: summary}

Table 1 highlights the effect of changing each parameter independently and the ratio with which each parameter can influence the dose. Primary and secondary beam projections were found to have the largest effect on the dose to the operator followed by the effect of changing the $x$-ray beam quality, while the effect of changing the source to image intensifier distance has the smallest effect.

\subsection{Results of the multiple regression analysis}

As mentioned earlier, two different regression models were applied for two different purposes. First, the results of the multiple polynomial regression model are presented in annex 1 together with the cubic equation that can be used to predict the ratio $R$ (Eq. 2) at any given combination of parameters. Second, the results of the random forest regression model are presented through the accuracy of the model and the resulted feature importance matrix. The accuracy of the model is shown in Fig. 14 where the predicted value is compared to the true value in the test data set. The overall validation score is $R 2=0.92$ which is reasonably good as most of the test samples fall within $\pm 25 \%$ error bounds. It means the model can be used to describe the importance of each feature. 
Fig. 15 shows that the beam projections (primary and secondary angles) have the highest impact on the dose in the resulted random forest regression model. Field size and beam quality share approximately similar importance on the dose to the operator. The impact of changing source to image intensifier distance (SID) seems to have lower impact on the dose compared to other parameters.

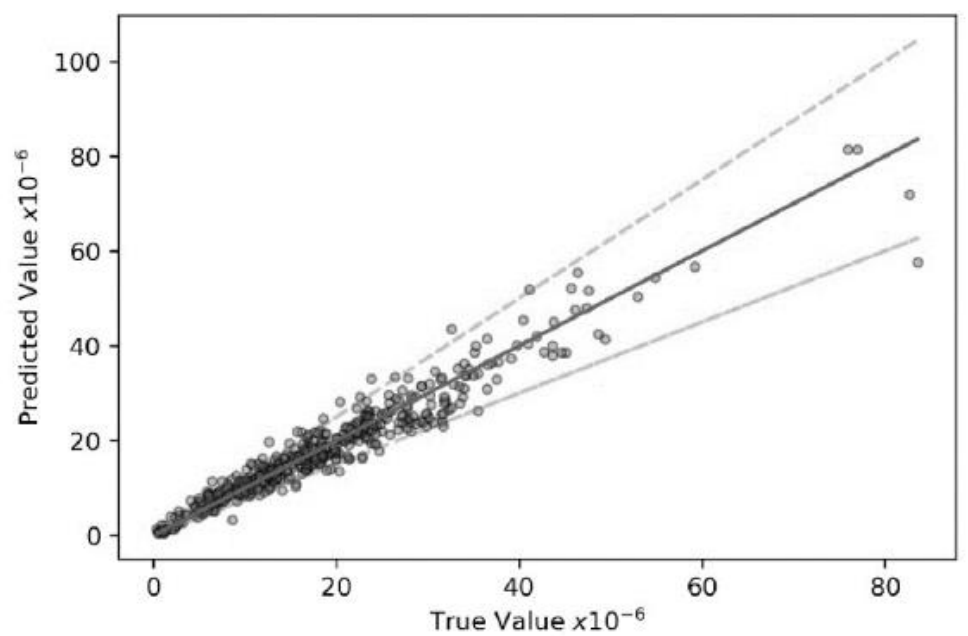

Fig. 14. Accuracy of the random forest regression model: predicted values vs. true values.

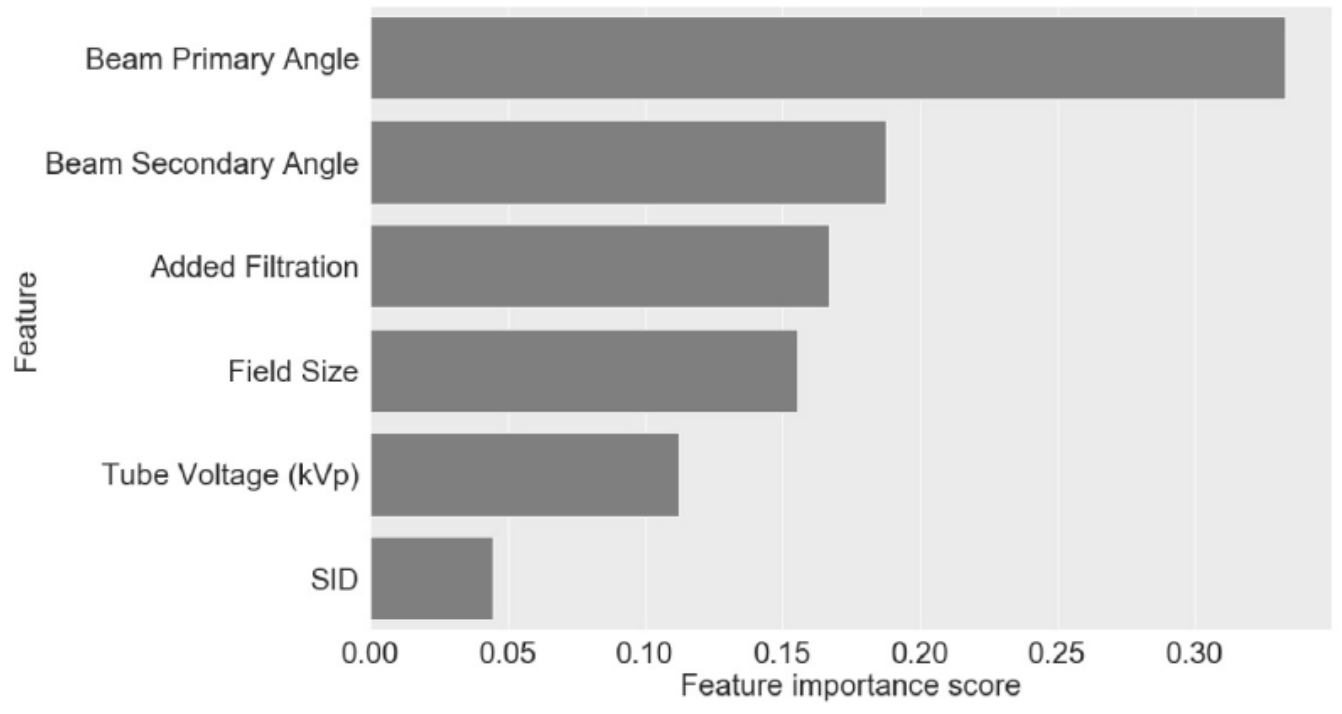

Fig. 15. Feature importance of the random forest regression of each studied parameter. 
Table 1. Effect of changing each studied parameter independently; Ratio between lowest and highest dose to the operator

\begin{tabular}{|c|c|c|c|c|c|c|c|}
\hline$k V p$ & $\begin{array}{c}\text { Primary } \\
\text { Angles }\end{array}$ & $\begin{array}{c}\text { Secondary } \\
\text { Angles }\end{array}$ & $\begin{array}{l}\text { Field Diameter } \\
\qquad(\mathrm{cm})\end{array}$ & $\begin{array}{l}\text { Added filtration } \\
\qquad(\mathrm{mm})\end{array}$ & $\begin{array}{l}\text { SID } \\
(\mathrm{cm})\end{array}$ & $\begin{array}{c}\mathrm{R}(\mathrm{Sv} / \mathrm{Gy}) \\
\frac{\text { operator } H_{p}(10)}{\text { Reference } K_{\text {air }}}\end{array}$ & $\begin{array}{c}\text { Ratio } \\
\text { (Max. /Min.) }\end{array}$ \\
\hline \multicolumn{8}{|c|}{ Effect of kVp } \\
\hline 70 & 0 & 0 & 20 & 0 & 100 & $3.53 \times 10^{-6}$ & \multirow{2}{*}{3.3} \\
\hline 120 & 0 & 0 & 20 & 0 & 100 & $1.17 \times 10^{-5}$ & \\
\hline \multicolumn{8}{|c|}{ Effect of Primary Angle } \\
\hline 70 & -45 & 0 & 20 & 0 & 100 & $2.45 \times 10^{-6}$ & \multirow{2}{*}{6.8} \\
\hline 70 & 75 & 0 & 20 & 0 & 100 & $1.65 \times 10^{-5}$ & \\
\hline 120 & -45 & 0 & 20 & 0 & 100 & $9.12 \times 10^{-6}$ & \multirow{2}{*}{3.5} \\
\hline 120 & 75 & 0 & 20 & 0 & 100 & $3.22 \times 10^{-5}$ & \\
\hline \multicolumn{8}{|c|}{ Effect of Secondary Angle } \\
\hline 70 & 0 & -30 & 20 & 0 & 105 & $4.55 \times 10^{-7}$ & \multirow{2}{*}{8.4} \\
\hline 70 & 0 & 15 & 20 & 0 & 105 & $3.82 \times 10^{-6}$ & \\
\hline 120 & 0 & -30 & 20 & 0 & 105 & $1.56 \times 10^{-6}$ & \multirow{2}{*}{8.6} \\
\hline 120 & 0 & 15 & 20 & 0 & 105 & $1.33 \times 10^{-5}$ & \\
\hline \multicolumn{8}{|c|}{ Effect of Field Diameter } \\
\hline 70 & 0 & 0 & 20 & 0 & 100 & $3.53 \times 10^{-6}$ & \multirow{2}{*}{2.1} \\
\hline 70 & 0 & 0 & 30 & 0 & 100 & $7.38 \times 10^{-6}$ & \\
\hline 120 & 0 & 0 & 20 & 0 & 100 & $1.17 \times 10^{-5}$ & \multirow{2}{*}{2.6} \\
\hline 120 & 0 & 0 & 30 & 0 & 100 & $3.01 \times 10^{-5}$ & \\
\hline \multicolumn{8}{|c|}{ Effect of Added Copper Filtration } \\
\hline 70 & 0 & 0 & 20 & 0 & 100 & $3.53 \times 10^{-6}$ & \multirow{2}{*}{4.3} \\
\hline 70 & 0 & 0 & 20 & 0.9 & 100 & $1.52 \times 10^{-5}$ & \\
\hline 120 & 0 & 0 & 20 & 0 & 100 & $1.17 \times 10^{-5}$ & \multirow{2}{*}{2.6} \\
\hline 120 & 0 & 0 & 20 & 0.9 & 100 & $3.08 \times 10^{-5}$ & \\
\hline \multicolumn{8}{|c|}{ Effect of Source-to-Intensifier Distance } \\
\hline 70 & 0 & 0 & 20 & 0 & 100 & $3.53 \times 10^{-6}$ & \multirow{2}{*}{1.4} \\
\hline 70 & 0 & 0 & 20 & 0 & 120 & $2.40 \times 10^{-6}$ & \\
\hline 120 & 0 & 0 & 20 & 0 & 100 & $1.17 \times 10^{-5}$ & \multirow{2}{*}{1.2} \\
\hline 120 & 0 & 0 & 20 & 0 & 120 & $9.10 \times 10^{-6}$ & \\
\hline
\end{tabular}

\section{Discussion}

Several studies have examined the influence of some imaging conditions on the occupational exposure in interventional radiology. We evaluated the effect of several parameters extended over the operating range of IR procedures. The study of Schueler et al. [9] evaluated the ratio between air kerma measured at the dosimeter position and the KAP (Gy/Gy. cm2). In their findings, the ratio is proportional to the change in the beam field of view as such increasing the field of view from 20 to $28 \mathrm{~cm}$ increases the ratio by more than a factor of 1.5 for specific imaging conditions ( $80 \mathrm{kV}$ and $3.4 \mathrm{~mm} \mathrm{Al}$ ). In our study and for comparable imaging conditions, increasing the field of view from 20 to $30 \mathrm{~cm}$ increases the ratio $\mathrm{R}$ (Sv/Gy. $\mathrm{cm} 2$ ) by a factor of 2.5. Moreover, the study showed that by adding $0.2 \mathrm{~mm}$ copper filtration, the ratio (Gy/Gy. $\mathrm{cm} 2$ ) increases by 1.6 times, while, in our study, R (Sv/Gy. cm2) was almost doubled for comparable beam parameters. This difference can be attributed to the difference in dosimeter position and the patient thickness in the two models. Our study shows that the dose is directly proportional to both field size and $\mathrm{kVp}$, which is in agreement with the study of Koukorava et al. [10] and the study of Marshal et al. [31]. 
Regarding beam projections, we found that the LAO projections (tube closer to the operator) results in higher doses than the RAO projection; while, the cranial projections present significantly higher doses compared to caudal projections. This finding is in agreement with the study of Koukorava et al. [10] and Carinou et al. [11]. The study of Ferrari et al. 2018 and 2019 $[12,13]$ evaluated the ratio $\mathrm{Hp}(10) / \mathrm{KAP}\left(\mathrm{Sv} / \mathrm{Gy} . \mathrm{cm}\right.$ ) for mainly 4 projections (PA, $25^{\circ} \mathrm{LAO}, 25^{\circ}$ RAO and $25^{\circ} \mathrm{CRA}$ ) and 10 different beam qualities. However, the results cannot be directly compared to our results, as the location of the operator in both studies is considerably different. The operator phantom in the model of [13] was placed near the patient's abdomen with the hands almost in the $\mathrm{x}$-ray field that led to the dosimeter being located in front of the image intensifier. This location could occur also during jugular/subclavian venous access procedures that deemed to be uncommon in practice [32]. While in our model, the dosimeter was placed at $(52.5,-20$, and $49.5 \mathrm{~cm})$ from isocenter simulating typical operator position during procedures of femoral/radial access. Accordingly, by comparing the results of both studies for the four different beam projections, differences of a factor of 10 are found between the two locations, except for $25^{\circ}$ RAO. In that case, the position of the operator behind the image intensifier makes the shielding effect prominent in case of $25^{\circ}$ RAO compared to PA, $25^{\circ}$ LAO and $25^{\circ} \mathrm{CRA}$. In contrast, the variation of the ratio (Sv/Gy. $\mathrm{cm} 2$ ) at the location of the operator towards the patient pelvic area in the present study is insignificant across the four projections.

Thus, the relationship between the dose and different combinations of primary and secondary projections is complex and depends mainly on the location of the interventionalist relative to the beam isocenter. In any case, the study shows that is preferable, if possible, to use mild projections and to avoid hard angulations to reduce the dose to the staff in IR. However, in some clinical situations, it is inevitable for the interventionalist to use beam rotations to avoid irradiating the same part of skin of the patient as per the guidelines of patient dose optimization in practice.

The present study also provides the following model by which the scatter fraction $\mathrm{R}$ can be assessed for any given combination of $\mathrm{x}$-ray beam settings within the range studied here:

$$
R(S v / G y)=\left(\left(\sum_{i=0}^{83} T_{i} \beta_{i}\right)-55.77\right) \times 10^{-6}
$$

where $T$ is a term of the cubic equation as given in Appendix $A$ and is the corresponding coefficient as listed in Table A.2. This model can be used by IR practitioners to derive absorbed doses (by multiplying $\mathrm{R}$ by the dose reading of the KAP meter reported in the RDSR of a given irradiation event). The practitioner then can compare the exposure at different beam settings and beam projections, which allow them to evaluate their practice from a radiation protection point of view. Polynomial regression and random forests regression are known to cease working outside the interval in which it is defined or trained. Therefore, this predictive model is valid only within the intervals of the studied parameters and cannot be extrapolated outside those ranges. However, the study here covered a wide range of settings within the operating range in IR; thus, the model is expected to be valid in most of the cases. It is worth mentioning that this absorbed dose is an approximation of the operational quantity $\mathrm{Hp}(10)$ as no phantom for the operator was used in the simulations. The contribution of the backscatter component in this set-up is usually in the order of $10 \%$. In the future, the model could be adapted to calculate $\mathrm{Hp}(10)$ by introducing a correction factor that accounts for the backscatter component at different settings. However, such correction factor would not change the conclusion and the trends reported in this paper, as it is a quasi constant.

\section{Conclusion}


The study of the effect of several parameters that affect staff doses at a typical position of the main operator in IR was performed using Monte-Carlo simulations. In this study, we investigated the influence of theangiography beam settings while fixing the other non-controllable clinical variables. The impact of changing each parameter of the beam settingsindependently and simultaneously was studied. When each parameter changes independently, oblique projections primary andsecondary can change the scatter fraction by seven folds, while the beam quality $\mathrm{kVp}$ and added copper filtration - can change it by approximately 4 times each. The scatter fraction is significantly higher when using primary projections higher than $30^{\circ} \mathrm{LAO}$. The highest exposure occurs at $75^{\circ} \mathrm{LAO}$ combined with $30^{\circ} \mathrm{CRA}$. While mild primary projections within $30^{\circ}$ LAO to $30^{\circ}$ LAO moderately affect the dose to the operator.

Comparing the results with data in literature shows that the effect of the beam projection on the dose is strongly dependant on the position of the operator relative to the isocenter. The dose to the operator only slightly increases with the source to image intensifier distance. In addition, the results show complex relationships when different variables simultaneously change as such the contribution of each parameter to the resulted dose to the operator can be determined using a model ensembling approach such as random forest regression. Accordingly, the results of the random forest regression analysis showed that the contribution of the primary beam projection dominates the change in the operator dose, followed by the contribution of beam secondary projection, field diameter, $\mathrm{kVp}$ and added copper filtration. The study provides a predictive model, which can be used to assess the staff dose for any combination of $x$-ray beam settings within the range studied here. Finally, in the highlights of results, the study could be used for training the staff in interventional radiology to improve their practice by using optimum beam settings, which can help reducing their occupationalexposure. Other non-clinical variables such as operator position, dosemeter location, shielding position and orientation, and patient thickness can also affect the dose to the staff in IR. For that reason, another study will follow to study the influence of these parameters.

\section{Acknowledgements}

PODIUM project is funded by the 'CONCERT- European Joint Programme for the Integration of Radiation Protection Research 2014- 2018' under grant agreement No. 662287.

\section{References}

[1] Miller DL. Overview of contemporary interventional fluoroscopy procedures. Health Phys 2008. https://doi.org/10.1097/01.HP.0000326341.86359.0b.

[2] Vano E, Gonzalez L, Fernández JM, Haskal ZJ. Eye lens exposure to radiation in interventional suites: Caution is warranted. Radiology 2008. https://doi.org/10.1148/radiol.2482071800.

[3] Kim KP, Miller DL, Balter S, Kleinerman RA, Linet MS, Kwon D, Simon SL. Occupational radiation doses to operators performing cardiac catheterization procedures 2008. https://doi.org/10.1097/01.HP.0000290614.76386.35.

[4] Huda W, Abrahams RB. Radiographic techniques, contrast, and noise in x-ray imaging. AJR Am J Roentgenol 2015. https://doi.org/10.2214/AJR.14.13116.

[5] Quinn B, C. Radiography in the digital age: physics-exposure-radiation biology; 2011. ISBN 9780398086466. DOI: 10.1148/radiol.13134005.

[6] Schueler BA. Clinical applications of basic x-ray physics principles. RadioGraphics 1998. https://doi.org/10.1148/radiographics.18.3.9599394.

[7] General principles for the radiation protection of workers. Annals of the ICRP; 1997. 10.1016/S0146-6453(97)88275-9.

[8] Järvinen $H$, Buls N, Clerinx P, Jansen J, Miljanić S, Nikodemová D, Ranogajec-Komor $M$, D'Errico F. Overview of double dosimetry procedures for the determination of the effective dose to the interventional radiology staff 2008. https://doi.org/10.1093/rpd/ncn082. 
[9] Schueler BA, Vrieze TJ, Bjarnason H, Stanson AW. An investigation of operator exposure in interventional radiology. Radiographics 2006. https://doi.org/10. 1148/rg.265055127.

[10] Koukorava C, Carinou E, Ferrari P, Krim S, Struelens L. Study of the parameters affecting operator doses in interventional radiology using Monte Carlo simulations. Radiat Measur 2011. https://doi.org/10.1016/j.radmeas.2011.06.057.

[11] Carinou E, Ferrari P, Koukorava C, Krim S, Struelens L. Monte Carlo calculations on extremity and eye lens dosimetry for medical staff at interventional radiology procedures. Radiat Prot Dosimetry 2011. https://doi.org/10.1093/rpd/ncq573.

[12] Ferrari P, Bakhanova E, Becker F, Campani L, Chumak V, Jansen J et al. EURADOS Working Group 12 studies in interventional radiology for medical staff dosimetry. Nuovo Cimento della Societa Italiana di Fisica C 2018; 10.1393/ncc/i2018-18217-2.

[13] Ferrari P, Becker F, Jovanovic Z, Khan S, Bakhanova E, Principi S, Kristic D, Pierotti L, Mariotti F, Faj D, Turk T, Nikezic D, Bertolini M. Simulation of $\mathrm{Hp}(10)$ and effective dose received by the medical staff in interventional radiology procedures. J Radiol Prot 2019. https://doi.org/10.1088/1361-6498/ab2c42.

[14] Olgar T, Bor D, Berkmen G, Yazar T. Patient and staff doses for some complex x-ray examinations. J Radiol Prot 2009. https://doi.org/10.1088/0952-4746/29/3/004.

[15] Badal A, Zafar F, Dong H, Badano A. A real-time radiation dose monitoring system for patients and staff during interventional fluoroscopy using a GPU-accelerated Monte Carlo simulator and an automatic 3D localization system based on a depth camera. In: Medical imaging 2013: physics of medical imaging. ISBN 9780819494429; 2013: 10.1117/12.2008031.

[16] Abdelrahman M, Lombardo P, Vanhavere F, Seret A, Phillips C, Covens P. First steps towards online personal dosimetry using computational methods in interventional radiology: Operator's position tracking and simulation input generation. Radiat Phys Chem 2020. https://doi.org/10.1016/j.radphyschem.2020.108702.

[17] Podium: Personal online dosimetry using computational methods. URL:https://podiumconcerth2020.eu/.

[18] Draper NR, Smith H. Applied Regression Analysis. 3rd ed. 2014.

[19] Hernández G, Fernández F. A model of tungsten anode x-ray spectra. Med Phys 2016;10(1118/1):4955120.

[20] Hernández G, Fernández F. xpecgen: A program to calculate x-ray spectra generated in tungsten anodes. J Open Source Softw 2016. 10.21105/joss.00062.

[21] Pelowitz DB. MCNPX TM User's manual version 2.7.0; 2011. LA-CP-05-0369.

[22] Shultis JK, Faw RE. An MCNP primer. Structure 2006.

[23] Vanhavere F, Carinou E, Gualdrini G, Clairand I, Merce MS, Ginjaume M et al. ORAMED: optimization of radiation protection of medical staff; 2012. ISBN 978-3- 943701-01-2. DOI: 10.1111/j.1398-9995.2011.02748.x.

[24] Lombardo PA, Vanhavere F, Lebacq AL, Struelens L, Bogaerts R. Development and validation of the realistic anthropomorphic flexible (RAF) phantom. Health Phys 2018;114(5):489-99. https://doi.org/10.1097/HP.0000000000000805.

[25] Almén A, Abdelrahman M, Andersson M, Balcaza V, Camp A, Duch M et al. D9.113 - report from the feasibility study performed in two hospitals. CONCERT European Joint Programme for the Integration of Radiation Protection Research, H2020- 662287; 2019. URL: https://www.concert-

H2020.eu/Document.ashx?dt=web\&file=/Lists/Deliverables/Attachments/190/D9.113_\%20Re port\%20from\%20the\%20feasibility\%20study\%20performed\%20in\%20two\%20hospitals_appro ved08012020.pdf\&guid=01b5ac77-b2ec-4cda-9c98-917dba396f0f.

[26] Principi S, Farah J, Ferrari P, Carinou E, Clairand I, Ginjaume M. The influence of operator position, height and body orientation on eye lens dose in interventional radiology and cardiology: Monte Carlo simulations versus realistic clinical measurements. Physica Med 2016. https://doi.org/10.1016/j.ejmp.2016.08.010. 
[27] National Electrical Manufacturers Association. NEMA PS3/ISO 12052, Digital imaging and communications in medicine (DICOM). ???? URL:https://www.nema.org/Standards/Pages/Digital-Imaging-and-Communications-inMedicine.aspx.

[28] Pedregosa F, Varoquaux G, Gramfort A, Michel V, Thirion B, Grisel O, Blondel M, Prettenhofer P, Weiss R, Dubourg V, Vanderplas J, Passos A, Cournapeau D, Brucher, M, Perrot M, Duchesnay É. Scikit-learn, Machine learning in Python. J Mach Learn Res 2011. arXiv:1201.0490.

[29] Breiman L. Random forests. Mach Learn 2001. https://doi.org/10.1023/A:1010933404324. [30] Harvey HB, Sotardi ST. The Pareto Principle. J Am College of Radiol 2018. https://doi.org/10.1016/j.jacr.2018.02.026.

[31] Marshall NW, Faulkner K. The dependence of the scattered radiation dose to personnel on technique factors in diagnostic radiology. Br J Radiol 1992. https://doi.org/10.1259/0007-128565-769-44.

[32] Weil J. The usual vascular access. In: Cardiac catheterization for congenital heart disease: from fetal life to adulthood. Springer-Verlag Milan. ISBN 9788847056817; 2015. p. 159-169. DOI: 10.1007/978-88-470-5681-7_11.

\section{Appendix A. Multivariate regression model}

In this annex, a predictive model using multivariate polynomial regression is described. This model can help the user to assess the ratio $\mathrm{R}$ (Sv/Gy) at the given dosemeter location for any combination of $\mathrm{x}$-ray beam settings within the range studied in this paper. Our model was build using the implementation of polynomial regression in Scikit-learn [28]. The function correlates six independent variables $(\mathrm{X} 1: \mathrm{X} 6)$ to the dependent variable $(\mathrm{Y})$ where:

$\mathrm{X} 1$ : $\mathrm{x}$-ray tube peak voltage $(\mathrm{kVp})$

$\mathrm{X} 2$ : positioner primary angle in degrees (+ for LAO and - for RAO)

$\mathrm{X} 3$ : positioner secondary angle in degrees (+ for CRA and - for CAU)

$\mathrm{X} 4$ : added copper filtration thickness in $\mathrm{mm}$

$\mathrm{X}_{5}$ : field diameter in $\mathrm{cm}$

$X_{6}$ : source-to-image intensifier distance (SID) in $\mathrm{cm}$

Y: R (Sv/Gy)

Therefore, the fitted model yields the following formula to predict the ratio $\mathrm{R}\left(\frac{S v)}{G y}\right)$ :

$$
y=\left(\left(\sum_{i=0}^{83} T_{i} \beta_{i}\right)-55.77\right) \times 10^{-6}
$$

Where $T$ is a term of the cubic equation and $\beta$ is the corresponding coefficient as listed in table 1. 
Table A.2

List of $\beta$ coefficients of the quadratic equation of the fitted model.

\begin{tabular}{|c|c|c|c|c|c|c|c|c|}
\hline i & $\mathrm{T}$ & Coefficient & $\mathrm{i}$ & $\mathrm{T}$ & Coefficient & $\mathrm{i}$ & $\mathrm{T}$ & Coefficient \\
\hline 0 & 1 & $2.42 \mathrm{E}-06$ & 28 & $X_{1}^{3}$ & $1.49 \mathrm{E}-05$ & 56 & $X_{2} X_{3} X_{5}$ & $3.49 \mathrm{E}-05$ \\
\hline 1 & $X_{1}$ & $-5.42 \mathrm{E}-02$ & 29 & $X_{1}^{2} X_{2}$ & $-3.03 E-06$ & 57 & $X_{2} X_{3} X_{6}$ & $-3.10 \mathrm{E}-06$ \\
\hline 2 & $X_{2}$ & $-2.73 \mathrm{E}-04$ & 30 & $X_{1}^{2} X_{3}$ & $-3.16 \mathrm{E}-06$ & 58 & $X_{4}^{2} X_{2}$ & $-4.00 \mathrm{E}-01$ \\
\hline 3 & $x_{3}$ & $-2.94 \mathrm{E}-04$ & 31 & $X_{1}^{2} X_{4}$ & $-1.48 \mathrm{E}-03$ & 59 & $X_{2} X_{4} X_{5}$ & $6.70 \mathrm{E}-03$ \\
\hline 4 & $x_{4}$ & $1.10 \mathrm{E}-02$ & 32 & $x_{1}^{2} x_{5}$ & $-2.04 \mathrm{E}-05$ & 60 & $x_{2} X_{4} X_{6}$ & $2.46 \mathrm{E}-02$ \\
\hline 5 & $x_{5}$ & $5.09 \mathrm{E}-04$ & 33 & $X_{1}^{2} X_{6}$ & $-8.79 \mathrm{E}-05$ & 61 & $X_{5}^{2} X_{2}$ & $3.86 \mathrm{E}-04$ \\
\hline 6 & $x_{6}$ & $4.51 \mathrm{E}-05$ & 34 & $X_{2}^{2} X_{1}$ & $9.55 \mathrm{E}-06$ & 62 & $X_{2} X_{5} X_{6}$ & $-2.01 E-04$ \\
\hline 7 & $X_{1}^{2}$ & $3.49 \mathrm{E}-03$ & 35 & $X_{1} X_{2} X_{3}$ & $-1.27 \mathrm{E}-06$ & 63 & $X_{6}^{2} X_{2}$ & $1.69 \mathrm{E}-04$ \\
\hline 8 & $x_{1} x_{2}$ & $-6.64 \mathrm{E}-04$ & 36 & $X_{1} X_{2} X_{4}$ & $-1.96 \mathrm{E}-04$ & 64 & $X_{3}^{3}$ & $3.03 E-05$ \\
\hline 9 & $X_{1} X_{3}$ & $7.02 \mathrm{E}-03$ & 37 & $X_{1} X_{2} X_{5}$ & $1.13 \mathrm{E}-04$ & 65 & $X_{3}^{2} X_{4}$ & $-6.63 E-03$ \\
\hline 10 & $X_{1} X_{4}$ & $-2.63 \mathrm{E}+00$ & 38 & $X_{1} X_{2} X_{6}$ & $-8.08 \mathrm{E}-06$ & 66 & $X_{3}^{2} X_{5}$ & $-1.66 \mathrm{E}-04$ \\
\hline 11 & $X_{1} X_{5}$ & $-1.49 \mathrm{E}-01$ & 39 & $X_{3}^{2} X_{1}$ & $-3.54 \mathrm{E}-05$ & 67 & $X_{3}^{2} X_{6}$ & $-3.24 \mathrm{E}-05$ \\
\hline 12 & $X_{1} X_{6}$ & $2.27 \mathrm{E}-02$ & 40 & $X_{1} X_{3} X_{4}$ & $6.54 \mathrm{E}-03$ & 68 & $X_{4}^{2} X_{3}$ & $-1.12 \mathrm{E}+00$ \\
\hline 13 & $x_{2}^{2}$ & $-1.66 \mathrm{E}-02$ & 41 & $X_{1} X_{3} X_{5}$ & $3.98 \mathrm{E}-04$ & 69 & $X_{3} X_{4} X_{5}$ & $5.27 \mathrm{E}-02$ \\
\hline 14 & $x_{2} X_{3}$ & $-4.92 \mathrm{E}-09$ & 42 & $X_{1} X_{3} X_{6}$ & $-1.38 \mathrm{E}-04$ & 70 & $X_{3} X_{4} X_{6}$ & $5.28 \mathrm{E}-02$ \\
\hline 15 & $X_{2} X_{4}$ & $-2.38 \mathrm{E}+00$ & 43 & $X_{4}^{2} X_{1}$ & $-7.45 \mathrm{E}-01$ & 71 & $X_{5}^{2} X_{3}$ & $5.26 \mathrm{E}-04$ \\
\hline 16 & $X_{2} X_{5}$ & $-2.60 \mathrm{E}-03$ & 44 & $X_{1} X_{4} X_{G}$ & $4.77 \mathrm{E}-02$ & 72 & $X_{3} X_{6} X_{6}$ & $-4.90 \mathrm{E}-04$ \\
\hline 17 & $X_{2} X_{6}$ & $-1.43 \mathrm{E}-02$ & 45 & $X_{1} X_{4} X_{6}$ & $2.39 \mathrm{E}-02$ & 73 & $X_{6}^{2} X_{3}$ & $2.28 \mathrm{E}-04$ \\
\hline 18 & $x_{3}^{2}$ & $8.24 \mathrm{E}-03$ & 46 & $X_{5}^{2} X_{1}$ & $6.80 \mathrm{E}-04$ & 74 & $X_{4}^{3}$ & $1.95 \mathrm{E}+02$ \\
\hline 19 & $X_{3} X_{4}$ & $-6.29 \mathrm{E}+00$ & 47 & $X_{1} X_{5} X_{6}$ & $1.39 \mathrm{E}-03$ & 75 & $X_{4}^{2} X_{5}$ & $3.92 \mathrm{E}+00$ \\
\hline 20 & $X_{3} X_{5}$ & $-2.81 \mathrm{E}-03$ & 48 & $X_{6}^{2} X_{1}$ & $-1.46 \mathrm{E}-04$ & 76 & $X_{4}^{2} X_{6}$ & $-6.68 \mathrm{E}+00$ \\
\hline 21 & $X_{3} X_{6}$ & $-1.54 \mathrm{E}-02$ & 49 & $x_{2}^{3}$ & $3.23 \mathrm{E}-06$ & 77 & $X_{5}^{2} X_{4}$ & $-1.91 \mathrm{E}-01$ \\
\hline 22 & $X_{4}^{2}$ & $4.65 \mathrm{E}+02$ & 50 & $X_{2}^{2} X_{3}$ & $1.19 \mathrm{E}-05$ & 78 & $X_{4} X_{5} X_{6}$ & $1.17 \mathrm{E}-01$ \\
\hline 23 & $X_{4} X_{5}$ & $1.06 \mathrm{E}-01$ & 51 & $X_{2}^{2} X_{4}$ & $2.64 \mathrm{E}-03$ & 79 & $X_{6}^{2} X_{4}$ & $-1.89 \mathrm{E}-02$ \\
\hline 24 & $X_{4} X_{6}$ & $5.78 \mathrm{E}-01$ & 52 & $X_{2}^{2} X_{5}$ & $2.55 \mathrm{E}-04$ & 80 & $X_{5}^{3}$ & $1.09 \mathrm{E}-02$ \\
\hline 25 & $x_{5}^{2}$ & $1.27 \mathrm{E}-02$ & 53 & $x_{2}^{2} X_{6}$ & $1.22 \mathrm{E}-04$ & 81 & $X_{5}^{2} X_{6}$ & $-8.48 \mathrm{E}-03$ \\
\hline 26 & $X_{5} X_{6}$ & $2.71 \mathrm{E}-02$ & 54 & $X_{3}^{2} X_{2}$ & $1.96 \mathrm{E}-05$ & 82 & $X_{6}^{2} X_{5}$ & $1.72 \mathrm{E}-03$ \\
\hline 27 & $X_{6}^{2}$ & $4.95 E-03$ & 55 & $X_{2} X_{3} X_{4}$ & $-4.34 \mathrm{E}-04$ & 83 & $X_{6}^{3}$ & $-1.77 \mathrm{E}-04$ \\
\hline
\end{tabular}

Table A. 3

Testing the model for some given parameters

\begin{tabular}{lcccc}
\hline x-ray tube voltage & $120 \mathrm{kVp}$ & $70 \mathrm{kVp}$ & $120 \mathrm{kVp}$ & $120 \mathrm{kVp}$ \\
Positioner primary angle & $0^{\circ}$ & $0^{\circ}$ & $15^{\circ}$ & $15^{\circ}$ \\
Positioner secondary angle & $0^{\circ}$ & $0^{\circ}$ & $15^{\circ}$ & $15^{\circ}$ \\
Added Copper filtration & 0.0 & 0.0 & $0.2 \mathrm{~mm}$ & 0.0 \\
Source to detector distance & $100 \mathrm{~cm}$ & $100 \mathrm{~cm}$ & $100 \mathrm{~cm}$ & $100 \mathrm{~cm}$ \\
Field diameter & $20 \mathrm{~cm}$ & $20 \mathrm{~cm}$ & $30 \mathrm{~cm}$ & $30 \mathrm{~cm}$ \\
Predicted $R(\mathbf{S v} / \mathrm{Gy})$ & $1.10 \mathrm{E}-05$ & $2.95 \mathrm{E}-06$ & $6.43 \mathrm{E}-05$ & $3.16 \mathrm{E}-05$ \\
Simulated $R(\mathbf{S v} / \mathrm{Gy})$ & $1.17 \mathrm{E}-05$ & $3.53 \mathrm{E}-06$ & $6.20 \mathrm{E}-05$ & $3.34 \mathrm{E}-05$ \\
\hline
\end{tabular}

